\title{
Einige Lösungen \\ der Einsteinschen Feldgleichungen mit idealer Flüssigkeit, die sich in einen fünfdimensionalen flachen Raum einbetten lassen
}

\author{
H. Stephani \\ Theoretisch-Physikalisches Institut der Universität Jena
}

Eingegangen am 1. März 1968

\begin{abstract}
All solutions of the Einstein equations for a perfect fluid are given, which are invariantly characterized by: embedding class one, Petrov type $D$, zero acceleration of matter. Among these solutions are inhomogeneous cosmological models and special solutions with spherical symmetry.
\end{abstract}

Ein Rechenfehler führte in einer früheren Arbeit [1] des Verfassers zu der Feststellung, daß es keine beschleunigungsfreien Lösungen (mit idealer Flüssigkeit) der Einbettungsklasse 1 und des Petrowtyps $D$ gibt. Wir wollen diese Lösungen jetzt explizit bestimmen; man vergleiche zum folgenden die oben zitierte Arbeit.

Wenn sich Lösungen der Einsteinschen Gleichungen mit idealer Flüssigkeit

$$
R_{i n}-\frac{1}{2} R g_{i n}=T_{i n}=(\mu+p) u_{i} u_{n}+p g_{i n}
$$

in einen fünfdimensionalen flachen Raum einbetten lassen, existiert ein symmetrischer Tensor $\Omega_{m n}$, aus dem man den Krümmungstensor bilden kann

$$
R_{i n k l}=e\left(\Omega_{i k} \Omega_{n l}-\Omega_{i l} \Omega_{k n}\right), \quad e= \pm 1
$$

und der den Gleichungen

$$
\Omega_{i n ; k}=\Omega_{i k ; n}
$$

genügt. Hat der Krümmungstensor den Typ $D\left(=I_{e}\right)$, so gilt

$$
\begin{aligned}
p & =C^{2}, \quad \mu=C(3 C+2 D), \quad e=+1 \\
\Omega_{i n} & =2 C u_{i} u_{n}+C g_{i n}+D v_{i} v_{n} \\
u_{i} u^{i} & =-1, \quad u_{i} v^{i}=0, \quad v_{i} v^{i}=1,
\end{aligned}
$$

und für beschleunigungsfreie Strömungen folgt aus (3) bei teilweiser Berücksichtigung von (2) das System

$$
\begin{aligned}
u_{m ; n} & =a_{1} v_{m} v_{n}+a_{2}\left(w_{m} w_{n}+z_{m} z_{n}\right) \\
v_{m ; n} & =a_{1} u_{m} v_{n}+a_{3} z_{m} v_{n}+a_{4} w_{m} v_{n} \\
C,_{m} & =2 C a_{2} u_{m} \\
D,_{m} & =\left[(2 C+D) a_{1}-2 C a_{2}\right] u_{m}+d v_{m}+C a_{3} z_{m}+C a_{4} w_{m} .
\end{aligned}
$$


Da die Vektorfelder $u_{i}$ und $v_{i}$ rotationsfrei sind und gleichzeitig auf die Normalform

$$
u^{i}=-u_{i}=(0,0,0,1), \quad v_{i}=(0,0, v, 0)
$$

gebracht werden können, kann man das Koordinatensystem (Ruhesystem der Flüssigkeit)

$$
\begin{aligned}
d s^{2} & =f^{2}(t)\left[d x^{2}+H^{2}(x, y) d y^{2}\right]+v^{2}(x, y, r, t) d r^{2}-d t^{2} \\
z_{i} & =(f, 0,0,0) ; \quad w_{i}=(0, f H, 0,0)
\end{aligned}
$$

einführen. Die Funktionen $f, H$ und $v$ lassen sich aus (2) und (5) bestimmen; man erhält zwei Lösungstypen:

$$
\begin{aligned}
d s^{2} & =b^{2} f^{2}\left(d x^{2}+\frac{\sinh ^{2} x}{\sin ^{2} x} d y^{2}\right)+v^{2} d r^{2}-d t^{2}, \quad b \geq 0 \\
f^{2}(t) & =t^{2} b^{-1}-e a b ; \quad e= \pm 1 ; \quad a, b=\mathrm{const} . \\
v(x, r, t) & =f(t) d_{1}(x) d_{2}(r)+d_{3}(r) \int \frac{d t}{f(t)}+d_{4}(r) \\
p(t) & =e a f^{-4} ; \quad \mu(x, r, t)=p+\frac{2 e}{b v f^{3}}\left[a b d_{1}(x) d_{2}(r)+e d_{3}(r) t\right] .
\end{aligned}
$$

und

$$
\begin{aligned}
d s^{2} & =t\left(d x^{2}+d y^{2}\right)+v^{2}(x, y, z, t) d z^{2}-d t^{2} \\
v & =t \sqrt{t} h_{1}+\sqrt{t}\left[h_{2}+\frac{3}{4} h_{1}\left(x^{2}+y^{2}\right)+h_{3} x+h_{4} y\right]+h_{5} \\
p(t) & =\frac{1}{4} t^{-2}, \quad \mu(x, y, z, t)=-\frac{4 h_{1} t \sqrt{t}+h_{5}}{2 t^{2} v}+3 p \\
h_{i} & =h_{i}(z)
\end{aligned}
$$

Die Lösungen (8) hat man wohl als inhomogene Kosmen zu deuten. Ein einfacher Spezialfall entsteht für $h_{1}=h_{3}=h_{4}=0$ :

$$
d s^{2}=t\left(d x^{2}+d y^{2}\right)+\left[\sqrt{t} h_{2}(z)+h_{5}(z)\right]^{2} d z^{2}-d t^{2} .
$$

Die Unterräume $t=$ const. sind flach; der Druck hängt nur von der Zeit, die Ruhemassendichte auch von $z$ ab. Für große Zeiten geht dieser Kosmos gegen einen Lichtkosmos $\mu=3 p$.

Bei der zweiten Lösungsklasse (9) handelt es sich für $b<0$ und $d_{1}=0$ um spezielle kugelsymmetrische Räume.

Herrn Prof. Dr. Schmutzer und allen Mitgliedern der Arbeitsgruppe Relativitätstheorie danke ich für Anregungen und Diskussionen.

\section{Literatur}

1. Stephani, H.: Commun. Math. Phys. 4, 137 (1967).

H. STEPHANI

Theoretisch-Physikalisches Institut der Friedrich-Schiller-Universität Jena X-69 Jena, Max-Wien-Platz 1 